\title{
ISOLATION AND IDENTIFICATION OF SECONDARY METABOLITES OF BLACK BETEL (Piper betle L. var Nigra)
}

\author{
${ }^{1}$ Junairiah*, ${ }^{1}$ Ni'matuzahroh, ${ }^{1}$ Nabilah Istighfari Zuraidassanaaz, ${ }^{2}$ Lilis Sulistyorini \\ ${ }^{1}$ Department of Biology, Faculty of Science and Technology, Airlangga University, Mulyorejo 60115 \\ Surabaya, Indonesia \\ ${ }^{2}$ Faculty of Public Healthy, Airlangga University, Mulyorejo 60115 Surabaya, Indonesia \\ *E-mail: alip.jun1@gmail.com
}

Received 20 October 2018

Accepted 20 December 2018

\begin{abstract}
Piper betle L. var Nigra commonly known as black betel have a potential as drug raw materials. The leave of black betel can be used to resist bleeding so as to accelerate the healing of wounds on the skin, sputum, other uses are for epistaxis, as well as for dialysis, asthma, bronchitis, cough, and high blood pressure. Many uses of black betel leaves make it interesting to learn its metabolites compounds by phytochemical screening. So, this research aimed to isolate and identificate secondary metabolites of Piper betle L. var Nigra. This research used three kind of organic solvents, there were methanol, ethyl acetate, and n-hexane. Several screening tests were used to isolate and identificate the phytochemical compound, including screening of alkaloids, terpenoids/steroids, flavonoids, polyphenols, tannins, and saponins. The results showed that methanol extract of black betel leaves contained of alkaloids, terpenoids/steroid, flavonoids, polyphenols and tannins compounds. The ethyl acetate extract of black betel leaves contained of terpenoids/steroid, flavonoids and tannins compounds. The n-hexane extract of black betel leaves contained of terpenoids/steroid. The methanol extract of Piper betle L. var Nigra contained more secondary metabolites than n-hexane and ethyl acetate extracts.
\end{abstract}

Key words : methanol ; Phytochemical screening ; Piper betle L. var Nigra.

\section{Introduction}

Piper is one of the genera of the familia Piperaceae. Piperaceae is one of the largest angiosperm families, with 1050 species divided into three genera in Asia (Suwanphakdee et al., 2016). In Indonesia there is a black betel (Piper betle L. var Nigra), The leaves were reported to resist bleeding so as to accelerate the healing of wounds on the skin, sputum, other uses are for epistaxis, as well as for dialysis, asthma, bronchitis, cough, and high blood pressure (Moeljanto dan Mulyono, 2004). Black betel is a semi-woody dioecious creeper, the colour of stems are blackish red and the colour of leaves are blackish green. Falling leaf will leave a ring-shaped marks on the stem, the leaves have a distinctive aromatic odor and it is rapidly growing 5 up to 15 meters (Abdullah, 2011). It is distributed in the eastern part of the coast of Africa, Zanzibar island, Bonin island, Fuji islands, and the Indonesian islands (Moeljanto dan Mulyono, 2004). It is one of the important plants in the Asiatic region after coffee and tea respectively in terms of daily consumption (Kumar et al., 2009). Lots of black betel varieties in various countries, Piper betle L. var Nigra is one of endemic species of Indonesia.

Piper betle L. var Nigra has been known as ornamental plants and herbal remedies. 
Plants are recognized as aromatic as well as source of medicine. The extracts obtained from various plant parts possess medicinal properties and are used as colouring agent, preservative, sweetening agent and as an additive in many medicinal formulations. Plants restrain abundant amount of secondary metabolites, they are considered to be principal source of therapeutically active compounds (Craker, 2007). However, secondary metabolites of n-hexane, ethyl acetate and methanol extract of black betel leaf still remain to be investigated. So this research aimed to determine the secondary metabolites of n-hexane ethyl acetate and methanol extract of black betel leaves.

\section{Material and Methods}

\section{Plant Materials}

The whole plants of Piper betle L. var Nigra were collected from the Kayon flowers market, Surabaya, East Java, Indonesia.

\section{Extraction}

All of the black betel leaves were washed with running water to separate it from the rest of the land attached, then all of them were dried, after which the black betel leaves in small pieces like powder and inserted into each bottle containing of n-hexane, ethyl acetate, and methanol solvent. After two days, the extract solution of each black betel was separated from the pulp using filter paper.

\section{Screening of Alkaloids and Terpenoids / Steroids}

To screen alkaloids, 0.2 gram sample was dissolved in $4 \mathrm{~mL}$ of $96 \%$ ethanol using an ultrasonic vibrator. Then, $4 \mu \mathrm{l}$ extract was spotted on TLC plates. Kiesel gel GF 254 was used in stationary phase, chloroformmethanol (9:1) was used in mobile phase and Dragendorf reagent was used in color stain.
Orange color indicated the alkaloids in the extract.

To screen terpenoids/steroids, 0.3 gram sample was dissolved in $15 \mathrm{~mL}$ of ethanol, and then divided into three parts, each part was $5 \mathrm{~mL}$, referred to as a solution of II-A, II-B, and II-C. Then, it was identificated by TLC. $0.2 \mathrm{~g}$ sample was dissolved in $4 \mathrm{~mL}$ of n-hexane. $2 \mu \mathrm{l}$ extract was spotted on the stationary phase. Kiesel gel GF 254 was used as stationary phase, n-hexane - ethyl acetate (4:1) was used as mobile phase and anisaldehyde sulfuric acid was used as color stain. The existence of terpenoids/steroid was indicated by the occurrence of redpurple or purple. For Salkowski test, solution II-A is used as a blanco, $5 \mathrm{~mL}$ solution II-C was added 1-2 mL concentrated $\mathrm{H}_{2} \mathrm{SO}_{4}$ through the wall of the tube. The presence of unsaturated steroids was characterized by the emergence of the red color ring.

\section{Screening of Flavonoids and Polyphenols}

To screen flavonoids, $0.2 \mathrm{~g}$ sample was dissolved in $10 \mathrm{~mL}$ of $96 \%$ ethanol using an ultrasonic vibrator. Then, TLC testing method was used. $25 \mu$ l extract was spotted on a TLC plate. This TLC test used a thin layer of Kiesel Gel GF 254 in stationary phase, chloroform - methanol (9:1) in mobile phase, and $\mathrm{FeCl}_{3} 2 \%$ as color stains. Color spot was observed by UV $366 \mathrm{~nm}$ and $254 \mathrm{~nm}$. The presence of flavonoids was showed by spotting brown to black with $\mathrm{FeCl}_{3} 2 \%$ as a color stains.

To screen polyphenols, TLC testing method was used. $5 \mu l$ extract was spotted on a TLC plate. This TLC test used a thin layer of Kiesel Gel GF 254 in stationary phase, ethyl acetate - methanol - formic acid (16:4:1) as mobile phase, and $\mathrm{FeCl}_{3} 2 \%$ as color stain. Color spot was observed by UV $366 \mathrm{~nm}$ and $254 \mathrm{~nm}$. The presence of polyphenols was showed by brown to black spot with $\mathrm{FeCl}_{3} 2 \%$ as a color stains. 


\section{Screening of Tannins and Saponins}

To Screen Tannins, three tubes were provided. They were tube A (for blanco), tube $\mathrm{B}$ (for comparison tannins) and tube $\mathrm{C}$ (for samples of guava leaf extract). A tube contained $5 \mathrm{~mL}$ water, the tube $\mathrm{B}$ contained tannin reference solution in $5 \mathrm{~mL}$ water, and tube $\mathrm{C}$ contained guava leaf extract plus 5 $\mathrm{mL}$ water. Then each tube was added with 1 mL Folin solution, vortex 1 minute, let stand for 5 minutes. Then $2 \mathrm{~mL}$ sodium carbonate was added, vortex 1 minute, let stand for 10 minutes. Blue color indicated content of tannin.

To screen saponins, Forth test was used, $0.25 \mathrm{~g}$ sample was dissolved in $10 \mathrm{~mL}$ of boiling water and then centrifuged at 5000 rpm. The supernatant was taken as much as $10 \mathrm{~mL}$ and strongly shaked for 30 seconds. Positive result of forth test was showed by stable forth height at $1-10 \mathrm{~cm}$ above the surface for more than 30 minutes and when it was added by 1 drop of $2 \mathrm{~N}$ hydrochloric acid, the forth did not disappear. Then Liebermanm-Burchard test was used, using a solution of II-A and II-B test group of terpenoids and steroids solution II-A is used as a blanco. 3 drops of acetic acid anhydride and 1 drop of concentrated $\mathrm{H}_{2} \mathrm{SO}_{4}$ was added to $5 \mathrm{mI}$ II-B solution and shaked slowly. The blue green color occurred showed the presence of steroid saponins, red-purple color showed the presence of saponintriterpenoids and light yellow color indicates saturated saponin/sapogenin.

\section{Results and Discussion}

Screening of Alkaloids and Terpenoids/Steroids

The emergence of an orange stain on TLC test showed the presence of alkaloids compounds. In the Fig. 3(a) showed that methanol extract of black betel was stained orange, whereas the samples of ethyl acetate (Fig. 2(a)) and n-hexane (Fig. 1(a)) extracts were not stained orange stain. The emergence of red-purple/purple on the TLC test showed a group of terpenoids/steroid compounds. In the image shown that methanol, ethyl acetate, and n-hexane extract of black betel leaves were stained purplish red (Fig. 1(b), 2(b), and 3(b)). The emergence of the red ring on Salkowski test showed the presence of unsaturated steroids compounds. In Fig. 4 red ring was not showed on methanol, ethyl acetate, and nhexane extract of black betel leaves.

Screening of Flavonoids and Polyphenols

The emergence of brown to blackish stain on TLC test showed a flavonoid compound. In Fig. 3(c) and 2(c) showed that methanol and ethyl acetate extracts of black betel leaves were stained brown, meanwhile the nhexane extract of black betel leaves were not stained brown (Fig. 1(c)). The emergence of brown to blackish stain on TLC test showed the presence of polyphenols compounds group. In Fig. 3(d) showed that methanol extract of black betel leaves was stained black, while the stain of ethyl acetate (Fig. 2(d)) and n-hexane (Fig. 1(d)) extract were not stained black. 


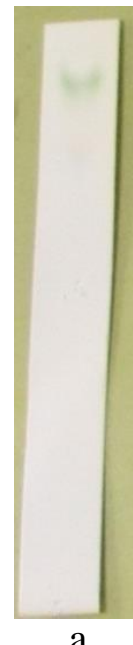

a

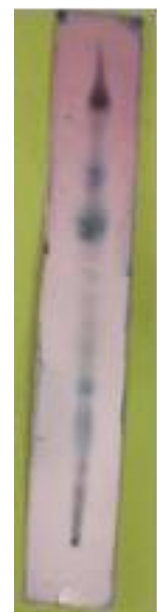

b

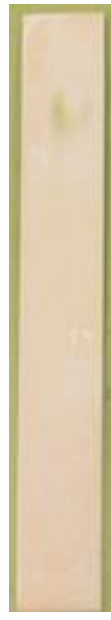

$\mathrm{C}$

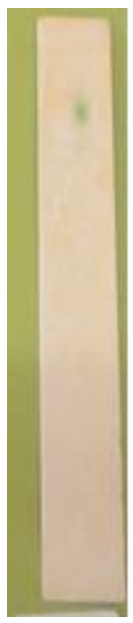

d

Figure 1. Phytochemical screening of n-hexane extract of black betel leaves (Piper betle L. var Nigra) with TLC method test. (a) Alkaloids, (b)Terpenoids/steroids, (c) Flavonoids, and (d) Polyphenols.

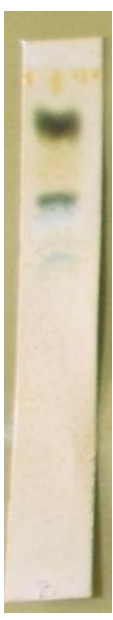

a

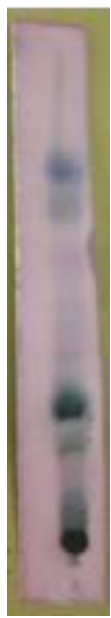

b

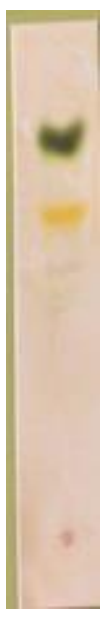

c

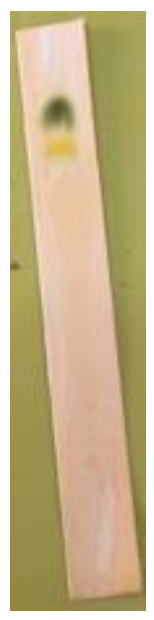

d

Figure 2. Phytochemical screening of ethyl acetate extract of black betel leaves (Piper betle L. var Nigra) with TLC method test. (a) Alkaloids, (b) Terpenoids/steroids, (c) Flavonoids, and (d) Polyphenols.

\section{Screening of Tannins and Saponins}

In Fig. 5 showed that the solutions were blue at methanol and ethyl acetate extract of black betel leave. It indicated the presence of tannin compounds. In screening of saponins, if the solution of extract showed forth in the forth test, it indicated the presence of saponins compounds. In Fig. 6 (a-c) showed that all of extract solutions (methanol, ethyl acetate, and n-hexane extract of black betel leave) did not produce a froth. Positive result in LiebermannBurchard test showed blue-green color, redpurple color and pale yellow color that indicated the presence of steroidal saponins, saponintriterpenoids and saturated sapogenin respectively. Meanwhile in this research, the methanol and ethyl acetate extract of black betel leaves showed green 
color and n-hexane extract showed clear

color (Fig. 6 (d), (f), (h)).

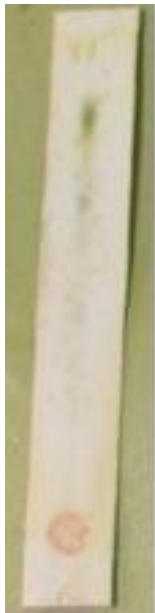

a

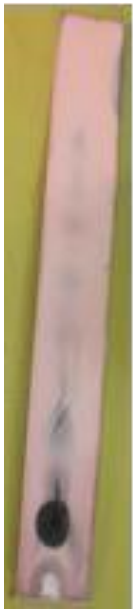

b

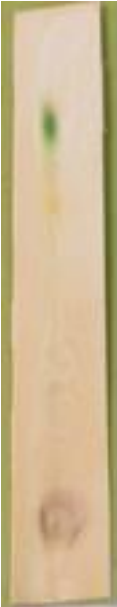

c

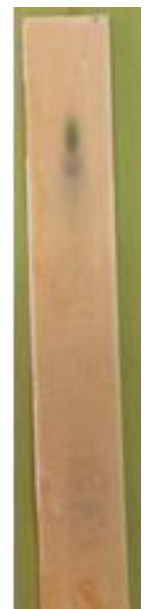

d

Figure 3. Phytochemical screening of methanol extract of black betel leaves (Piper betle L. var Nigra) with TLC method test. (a) Alkaloids, (b)Terpenoids/steroids, (c) Flavonoids, and (d) Polyphenols.

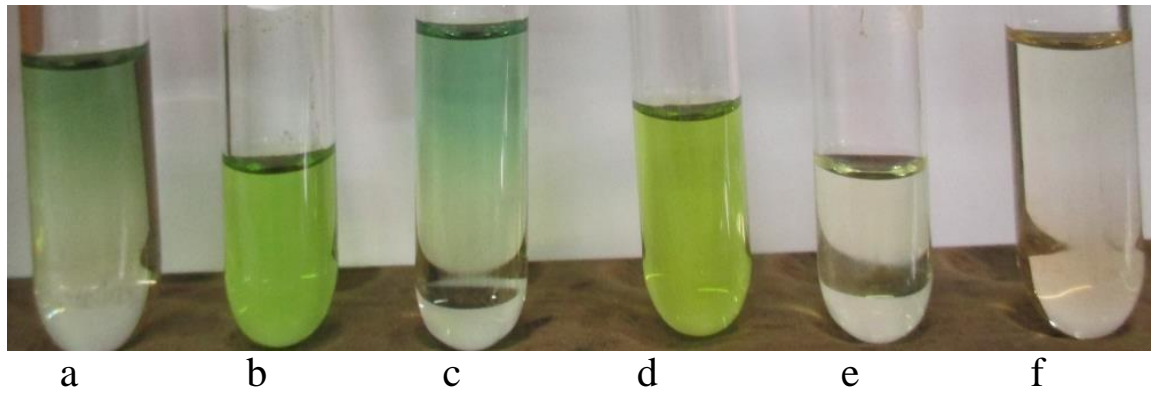

Figure 4. Screening of steroids using Salkowski test on various organic solvents extract of black betel leaves (Piper betle L. var Nigra). (a) Methanol extract of black betel (Piper betle L. var Nigra) + 1-2 mL of $\mathrm{H}_{2} \mathrm{SO}_{4}$ concentrated, (c) Ethyl acetate extract of black betel (Piper betle L. var Nigra) + 1-2 $\mathrm{mL}_{\text {of }} \mathrm{H}_{2} \mathrm{SO}_{4}$ concentrated, (e) $\mathrm{n}$-hexane extract of black betel (Piper betle L. var Nigra) + 1-2 mL of $\mathrm{H}_{2} \mathrm{SO}_{4}$ concentrated, and (b), (d), (f) blanco.

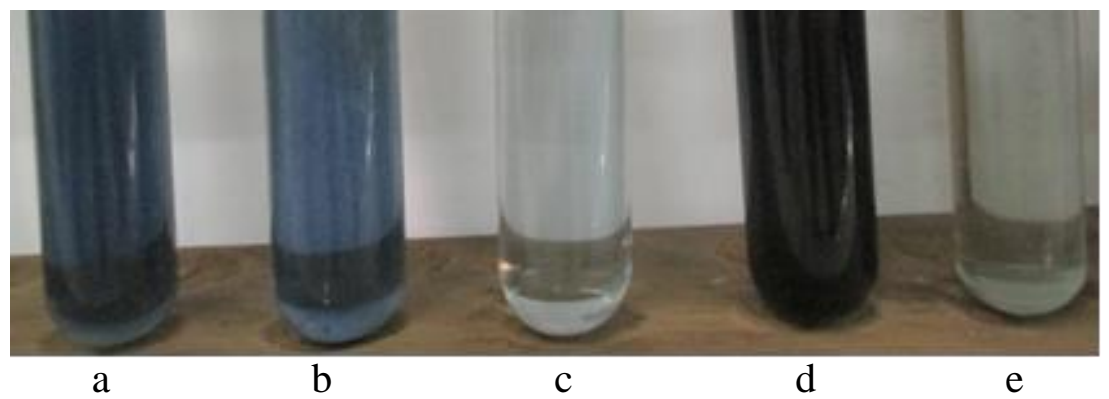

Figure 5. Screening of tannins on various organic solvents extract of black betel leaves (Piper betle L. var Nigra). (a) Methanol extract of black betel (Piper betle L. var Nigra), (b) Ethyl 
acetate extract of black betel (Piper betle L. var Nigra), (c) n-hexane extract of black betel (Piper betle L. var Nigra), (d) standard solution of tannins, and (e) blanco.
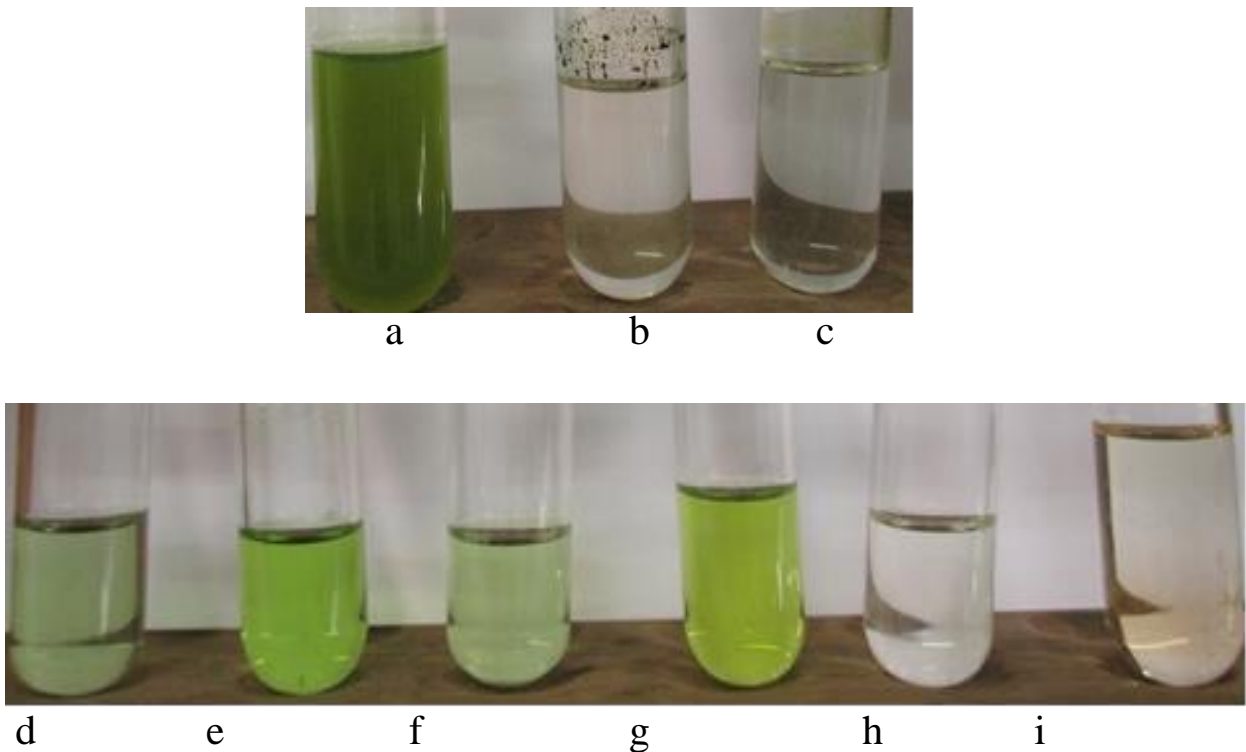

Figure 6. Screening of saponins on various organic solvents extract of black betel leaves (Piper betle L. var Nigra). (a) Forth test of methanol extract of black betel (Piper betle L. var Nigra), (b) Forth test of ethyl acetate extract of black betel (Piper betle L. var Nigra), (c) Forth test of n-hexane extract of black betel (Piper betle L. var Nigra), (d) Liebermann-Burchard test methanol extract of black betel (Piper betle L. var Nigra) +3 drops of acetic acid anhydride + 1 drop of concentrated $\mathrm{H}_{2} \mathrm{SO}_{4}$, (f) Liebermann-Burchard test ethyl acetate extract of black betel (Piper betle L. var Nigra) +3 drops of acetic acid anhydride +1 drop of concentrated $\mathrm{H}_{2} \mathrm{SO}_{4}$, (h) Liebermann-Burchard test n-hexane extract of black betel (Piper betle L. var Nigra) +3 drops of acetic acid anhydride +1 drop of concentrated $\mathrm{H}_{2} \mathrm{SO}_{4},(\mathrm{e}),(\mathrm{g})$, and (i) blanco.

Table 1. Identification of screening phytochemical in various organic solvents of extractblack betel leaves.

\begin{tabular}{|c|c|c|c|}
\hline \multirow{2}{*}{ Phytochemical } & \multicolumn{3}{|c|}{ Organic solvents of extract black betel leaves } \\
\cline { 2 - 4 } & n-hexane & Ethyl acetate & Methanol \\
\hline Alkaloids & - & - & + \\
\hline $\begin{array}{c}\text { Terpenoids/ } \\
\text { Steroids }\end{array}$ & + & + & + \\
\hline Flavonoids & - & + & + \\
\hline Polyphenols & - & - & + \\
\hline Tannins & - & + & + \\
\hline Saponins & - & - & - \\
\hline
\end{tabular}


Plant can be used as traditional medicine because it contain chemical compounds that have biological activity. The biologically active compound is a secondary metabolite which includes alkaloids, flavonoids, terpenoids, tannins and saponins. The content of secondary metabolite compounds in plant can be known by approaching method that provide information on the presence of secondary metabolite compounds. One of the method that can be used is the phytochemical screening method (Harbone, 1973). Before isolation and identification, the extraction was done first in this research. There are many extraction method, one of them is maseration.

After the black betel leaves was dried and mashed, then it divided into three equal parts to add three organic solvents to each part. Pulverization was useful to increase area surface of the particles contacted with solvent. After entering the powder, solvent exported the chemical compounds to be mixed with the substance of the attractants so that the process of escape can be more effective. The advantages of the maceration method are easy to perform, high enough rendamen product and low possiblility of damaged chemical compounds contained in the material because did not accompanied by giving heat. In table 1. showed that methanol extract of black betel leaves was contained of alkaloids, terpenoids/steroid, flavonoids, polyphenols and tannins compounds. The ethyl acetate extract of black betel leaves was contained of terpenoids/steroid, flavonoids and tannins compounds. The nhexane extract of black betel leaves was contained of terpenoids/steroid. In general, methanol solvents were used in the process of isolating organic compounds of natural materials because they could dissolve secondary metabolites (Prashant et al.,
2011). Methanol was a universal solvent which had a polar group (-OH) and a nonpolar group $(-\mathrm{CH})$ So that it could attract those analytes polar and nonpolar. There are several study about secondary metabolites, like Piper longum L. (Lee et al., 2001), Peperomia pellucida L., Marsilea quadrifolia L. (Jothi et al., 2013), Mentha longifolia L. (Al-bayati, 2009), and Euphorbia hirta L. (Basma et al., 2011).

Flavonoids and tannins are part of the phenolic compounds. Flavonoids that have an ortho-shaped hydroxy group will give intensive yellow fluorescence on UV366 when reacts with boric acid. Flavonoids have a variety of types and present in free form (aglikon) or bound as glycosides. The polymethoxyaglicone is nonpolar, the polyhydroxyaglycone is semi-polar, while the flavonoid glycoside is polar, because it contains a number of hydroxyl groups and sugar (Harbone, 1973). Therefore, the flavonoid group may be extracted in a universal methanol solvent. The tannins were shown after addition of $\mathrm{FeCl}_{3}$ which reacted with one of the hydroxyl groups on the tannin compound. $\mathrm{FeCl}_{3}$ addition produced a blackish-green color indicated the presence of a tannin condensed. Saponin is polar because it is a glycoside form of sapogenin. Saponin is active compound on the surface and cause foam in shaked water. The presence of forth on the saponin test indicated the presence of glycosides. Glycosides had the ability to form foam in water that was hydrolyzed to glucose and other compounds. The compound of saponins will tend to be extracted by semipolar solvents such as methanol. While, alkaloid screening had same principle with precipitation reaction that occurs due to the replacement of the ligand (Agostini-costa et al., 2012). 


\section{Acknowledgements}

Author would like to thank Ministry of

Research, Technology, and Higher

Education of Republic Indonesia, who

\section{References}

Abdullah MN. 2011. Daya Hambat Infusum Daun Sirih Terhadap Pertumbuhan Staphylococcus aureus yang Diisolasi dari Denture Stomatitis. Penelitian In Vitro. North Sumatra University.

Agostini-Costa TS, Vieira RF, Bizzo HR, Silveira D, Gimenes MA. 2012. Secondary Metabolites. Chromatography and Its Applications, Dr. Sasikumar Dhanarasu (Ed.). InTech. 131-164.

Al-bayati FA. 2009. Isolation and identification of antimicrobial compound from Mentha longifolia L. leaves grown wild in Iraq. Annals of Clinical Microbiology and Antimicrobials. 1-6.

Basma AA, Zakaria Z, Latha LY, Sasidharan S. 2011. Antioxidant activity and phytochemical screening of the methanol extracts of Euphorbia hirta L. Asian Pacific Journal of Tropical Medicine, 386-390.

Craker LE. 2007. Medicinal and aromatic plant e future opportunities. In: Janick J, Whipkey A, eds. Issues in New Crops and New Uses. VA: Alexandria ASHS Press. 248-257.

Harbone JB. 1973. Phytochemical Methods: A Guide to Modern Techniques of Plant Analysis. Springer Science and Business Media.

Jothi, Gini TG, Jeya G. 2013. Preliminary Phytochemical Screening of Whole funded this research via Distinguished Research Grant of Higher Education 2017, research contract No. 597/UN3.

14/LT/2017.

Plant Extracts of Peperomia pellucida (Linn.) HBK (Piperaceae) and Marsilea quadrifolia Linn. (Marsileaceae). International Journal of Pharmacognosy and Phytochemical Research, 5(3):200-214.

Kumar N, Misra P, Dube A, Bhattacharya S, Dikshit M, Ranade S. 2009. Piper betle L. a maligned Pan-Asiatic plant with an array of pharmacological activities and prospects for drug discovery. Curr Sci, 99:922-932.

Lee SE, Park BS, Kim MK, Choi WS, Kim HT, Cho KY, Lee SG, Lee HS. 2001. Antifungal Activity of Pipernonaline, a Piperidine Alkaloid Derived from Long Pepper, Piper longum L., against Phytopathogenic Fungi. Crop Prot. 20:523-528.

Moeljanto RD, Mulyono. 2004. Khasiat dan Manfaat Daun Sirih Obat Mujarab dari Masa ke Masa First Edition. Agromedia Pustaka.

Prashant, T., Bimlesh, K., Mandeep, K., Gurpreet, K., Harleen, K. 2011. Phytochemical screening and extraction: A Review. Internationale Pharmaceutica Sciencia. 1(1): 98-106.

Suwanphakdee C, Simpson DA, Hodkinson T, Chantaranothai P. 2016. Taxonomic notes on the genus Piper (Piperaceae). Nordic Journal of Botany, 1-14. 